7th International Workshop on Astronomy and

Relativistic Astrophysics (IWARA 2016)

International Journal of Modern Physics: Conference Series

Vol. 45 (2017) 1760021 (8 pages)

(C) The Author(s)

DOI: $10.1142 / S 2010194517600217$

\title{
A Family of Hierarchical Symplectic Maps for N-body Simulations with Post-Newtonian Corrections
}

\author{
Guilherme Gonçalves Ferrari \\ Instituto de Física \\ Universidade Federal do Rio Grande do Sul (UFRGS) \\ Av. Bento Gonçalves, 9500, 91501-970 Porto Alegre, RS, Brazil \\ gg.ferrari@gmail.com
}

Published 15 August 2017

\begin{abstract}
Symplectic maps are well known for preserving the phase-space volume in Hamiltonian dynamics and are particularly suited for problems that require long integration times, such as the $N$-body problem. However, when combined with a varying time-step scheme, they end up losing its symplecticity and become numerically inefficient. We address this problem by using a recursive Hamiltonian splitting based on the time-symmetric value of the individual time-steps required by the particles in the system. We present a family of 48 quasi-symplectic maps with different orders of convergence (2nd-, 4th\& 6th-order) and three time-stepping schemes: i) 16 using constant time-steps, ii) 16 using shared adaptive time-steps, and iii) 16 using hierarchical (individual) time-steps. All maps include post-Newtonian corrections up to order 3.5PN. We describe the method and present some details of the implementation.
\end{abstract}

Keywords: Symplectic Integrators, $N$-body Simulations, Post-Newtonian Corrections.

PACS numbers: $98.80 . J \mathrm{k}, 04.25$

\section{Introduction}

Historically, fully self-consistent realistic astrophysical $N$-body simulations have been impossible, either because of the lack of enough computational power or the absence of multi-physics simulation codes. On gravitational $N$-body codes the main bottleneck is often attributed to the amount of computational work needed to accurately evolve the orbits of the particles in the system on a star-by-star basis. Therefore, $N$-body simulations require both, a fast way to calculate the forces and an accurate integration method to evolve the particles in time.

In the last decade $N$-body codes have greatly benefited from the computing power of modern, highly parallel, devices such as multi-core CPUs, GPUs and

This is an Open Access article published by World Scientific Publishing Company. It is distributed under the terms of the Creative Commons Attribution 4.0 (CC-BY) License. Further distribution of this work is permitted, provided the original work is properly cited. 
FPGAs. Nowadays moderate sized $\left(N \sim 10^{3}-10^{4}\right) N$-body simulations are feasible on laptops or desktop computers equipped with such devices. To go on par with the increase in computing power of modern computers, there is a growing trend for multi-scale, multi-physics astrophysical simulations. Basically, the two main approaches for such endeavor can be described as follows: i) the monolithic approach consists in combine a diversity of astrophysical domains in a single numerical code; ii) the modular approach consists in develop specialized, single domain, standalone codes under the umbrella of a modular framework, which would act as a software layer responsible for the coupling of the different codes and astrophysical domains. Examples of codes employing such approaches are i) the Gadget ${ }^{1}$ code and ii) the $\mathrm{AMUSE}^{2}$ code, respectively.

The monolithic approach is most known for historical reasons and has been used in community codes since the 60's. However, it's been increasingly difficult to continue developing such codes, which demands from the programmer to be an specialist in all astrophysical domains and algorithms implemented in the code. For this very reason, the learning curve for any new astrophysicist, programmer or student interested in contributing with the development of such codes can be quite steep. Therefore, the main bottleneck in codes using the monolithic approach lies in the software development.

In the case of modular approaches, such intricacies are mostly avoided. Due to the modular design of such frameworks, the interested developer is free to contribute in any part of the software which best fits its area of specialty. This way, besides the character organizational of a modular approach, it facilitates the inclusion of new physics, the implementation of new algorithms, the comparison of different solvers for the same problem and it is more accessible to new developers and students who want to learn and/or contribute to the framework.

An important question that must be addressed during the development of a modular framework, though, is how to couple the different codes, which generally implement different astrophysical domains, algorithms and are possibly written in different programming languages. In the AMUSE code, the coupling is done by a hybrid method named BRIDGE and firstly introduced by Ref. 3, as a way to combine two different gravitational solvers in order to study the co-evolution of a star-cluster orbiting a parent galaxy.

The BRIDGE method is based on a 2nd-order extension of the mixed variable symplectic (MVS) scheme developed in the context of long term integrations of planetary systems. In its classical version, ${ }^{3}$ the BRIDGE method made possible the coupling of a highly accurate direct code with a fast tree-code in a single compound solver which can deal with the co-evolution of collisionless and collisional systems self-consistently. This BRIDGE method is quite powerful, for it allows to selfconsistently combine different specialized solvers without the necessity of modify each one of the solvers individually.

In Ref. 4 a recursive Hamiltonian split based on the time-step size of particles was proposed for the $N$-body problem. This method can be understood as a flavor 
of a divide-and-conquer algorithm, allowing the subdivision of the original problem into many smaller problems, each one defined by its own Hamiltonian function. In this way the original problem is self-consistently solved by solving and coupling each of the sub-problems throughout the recursive hierarchy.

In the present work we developed a generalization of the previous methods. We combine BRIDGE with the ideas introduced in Ref. 4 and construct a family of 48 quasi-symplectic maps with different orders of convergence (2nd-, 4th- \& 6th-order) and three time-stepping schemes: i) 16 using constant time-steps, ii) 16 using shared adaptive time-steps, and iii) 16 using hierarchical (individual) time-steps. In all cases we took special care to respect the time-symmetry of the maps which also includes post-Newtonian corrections up to order 3.5PN.

We give a brief review of the symplectic formalism in $\S 2$ and explain our timestep criterion in $\S 3$. In $\S 4$, we present a family of hierarchical symplectic maps, detailing how the post-Newtonian terms are included in a time-symmetric way. Finally, in $\S 5$ we give our conclusions.

\section{Symplectic Formalism}

Consider a Hamiltonian $H$ with canonical coordinates $(\mathbf{r}, \mathbf{p})$. The time evolution of an arbitrary quantity $\varrho \equiv \varrho(\mathbf{r}, \mathbf{p})$ can be described by the following equation,

$$
\frac{\partial \varrho}{\partial t}=\mathcal{H} \varrho,
$$

where $\mathcal{H}$ is the operator associated with the Hamiltonian, $H$, and $\mathcal{H} \varrho \equiv\{\varrho, \mathcal{H}\}$ with $\{$,$\} denoting the Poisson bracket. If there is no explicit time-dependency in$ the Hamiltonian function, the formal solution of Eq. 1 is given by,

$$
\varrho(t+\tau)=e^{\tau \mathcal{H}} \varrho(t) .
$$

In cases where we can split $\mathcal{H}$ into operators analytically solvable $\mathcal{T}$ and $\mathcal{U}$, such that $\mathcal{H}=\mathcal{T}+\mathcal{U}$, it is possible to decompose the time evolution of $\varrho$ in two parts, with $\mathcal{T}$ evolving only positions and $\mathcal{U}$ evolving only momenta, i.e.,

$$
\begin{aligned}
& e^{\tau \mathcal{T}} \varrho\left(\mathbf{r}_{0}, \mathbf{p}_{0}\right)=\varrho\left(\mathbf{r}_{0}+\tau \mathbf{v}_{0}, \mathbf{p}_{0}\right) \equiv \varrho\left(\mathbf{r}_{1}, \mathbf{p}_{0}\right), \\
& e^{\tau \mathcal{U}} \varrho\left(\mathbf{r}_{0}, \mathbf{p}_{0}\right)=\varrho\left(\mathbf{r}_{0}, \mathbf{p}_{0}+\tau \mathbf{F}_{0}\right) \equiv \varrho\left(\mathbf{r}_{0}, \mathbf{p}_{1}\right),
\end{aligned}
$$

where subscripts indicates the initial and final states of coordinates, $\mathbf{v}$ and $\mathbf{F}$ are the velocity and force, respectively, and $\tau$ is the time-step size. A 1st-order approximation to Eq. 2 can thus be written as,

$$
\phi_{\tau} \equiv e^{\tau \mathcal{U}} e^{\tau \mathcal{T}}=e^{\tau\left(\mathcal{T}+\mathcal{U}+\frac{\tau}{2}[\mathcal{U}, \mathcal{T}]+\mathcal{O}\left(\tau^{2}\right)\right)}=e^{\tau \tilde{\mathcal{H}}}
$$

which exactly evolves a surrogate Hamiltonian $\tilde{\mathcal{H}}=\mathcal{H}+\mathcal{H}^{\text {err }}$. The associated error $\mathcal{H}^{\text {err }}=\frac{\tau}{2}[\mathcal{U}, \mathcal{T}]+\mathcal{O}\left(\tau^{2}\right)$ is also Hamiltonian, meaning that, for small enough $\tau$, it remains bounded around its initial value. Here, [,] denotes a commutator. 
A 2nd-order approximation can be constructed as a composition of a 1st-order map and its self-adjoint as follows,

$$
\phi_{\tau}^{2} \equiv \phi_{\tau / 2}^{\dagger} \circ \phi_{\tau / 2}=e^{\frac{\tau}{2} \mathcal{T}} e^{\tau \mathcal{U}} e^{\frac{\tau}{2} \mathcal{T}}
$$

with the corresponding error given by

$$
\mathcal{H}_{2}^{\text {err }}=\frac{\tau^{2}}{12}\left([\mathcal{U},[\mathcal{U}, \mathcal{T}]]-\frac{1}{2}[\mathcal{T},[\mathcal{T}, \mathcal{U}]]\right)+\mathcal{O}\left(\tau^{4}\right) .
$$

In general, maps of order $n+2$ can be constructed by composition of maps of order $n$ according to the method of Ref. 5. Notice that because these maps are time-symmetric, only even-order maps exist.

As such, there are a series of advantages in using symplectic maps to evolve Hamiltonian systems, the most important ones being that they preserve the phasespace volume and that they are time-symmetric by construction. However, for highly stiff Hamiltonian problems it seems appropriated to adopt a varying time-step size for computational efficiency.

\section{Time-step Criterion}

Even though it is impossible ${ }^{\mathrm{a}}$ to preserve the symplecticity of the maps while varying the time-step size, one can still preserve its time-symmetric nature by a careful choice of the time-step function. In our case we compute $\tau$ in symmetrized way,

$$
\tau_{n+1 / 2}=\frac{\eta}{\omega_{n+1 / 2}}, \quad \omega_{n+1 / 2}^{2}=\frac{1}{2}\left[\omega_{n}^{2}+\omega_{n+1}^{2}\right]
$$

where $\eta$ is a parameter of precision for the integration and $\omega \equiv \omega(\mathbf{r}, \mathbf{p})$ is a time-step frequency function. For a gravitational system we use, for example, the following pairwise function,

$$
\omega_{i j}^{2}=\frac{1}{r_{i j}^{2}}\left(v_{i j}^{2}+\frac{2 \mu_{i j}}{r_{i j}}\right),
$$

where $\mu_{i j}$ is the reduced mass of the $i j$-pair of particles. Finally, in order to eliminate the character implicit of Eq. 8, we use instead a 1st-order Taylor expansion for $\omega_{n+1 / 2}^{2}$, such that,

$$
\omega_{n+1 / 2}^{2} \approx \omega_{n}^{2}+\frac{1}{2} \frac{\eta}{\omega_{n}} \frac{d \omega_{n}^{2}}{d t}+\mathcal{O}\left(\tau^{2}\right),
$$

which is enough to approximately guarantee the time-symmetric nature of the maps, since the errors in Eqs. 6 and 10 are of the same order.

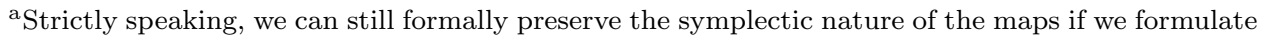
the problem in an extended phase-space where time and its associated momentum are evolved like the rest of canonical coordinates. This, however, is out of the scope of the present work. 


\section{Hierarchical Symplectic Maps}

In order to construct hierarchical symplectic maps, we now combine the ideas introduced in Refs. 3 and 4 as follows. First, we need a way to subdivide the original Hamiltonian problem into two disjoint sub-problems. This can be accomplished by using the Slow-Fast Hamiltonian split. ${ }^{4}$ In such case, the two sub-systems, $S$ and $F$, are defined by

$$
S \equiv\left\{i \in 1 \ldots N: \tau_{i} \geq \tau\right\}, \quad F \equiv\left\{i \in 1 \ldots N: \tau_{i}<\tau\right\}
$$

according to the time-symmetric time-step size required by each particle in the system. This way, the original Hamiltonian can be decomposed as follows:

$$
\begin{aligned}
\mathcal{H} & =\mathcal{H}_{S}+\left(\mathcal{U}_{S F}+\mathcal{H}_{F}\right), \\
& =\left(\mathcal{H}_{S}+\mathcal{U}_{S F}\right)+\mathcal{H}_{F}, \\
& =\mathcal{U}_{S F}+\left(\mathcal{H}_{S}+\mathcal{H}_{F}\right) .
\end{aligned}
$$

$\mathcal{H}_{S}$ and $\mathcal{H}_{F}$ are the Hamiltonian operators associated with sub-systems $S$ and $F$, and $\mathcal{U}_{S F}$ contains all the terms responsible for the coupling of $S$ and $F$. In Eq. 12, parenthesis were used to indicate three possible ways to couple the different subHamiltonians. By noticing that $\left[\mathcal{H}_{S}, \mathcal{H}_{F}\right]=0$, one can see that the third line in Eq. 12 is best suited for computational efficiency, since in this case sub-systems $S$ and $F$ can be solved in parallel. Thus, the map for such Hamiltonian decomposition can be written as

$$
\begin{aligned}
e^{\tau \mathcal{H}} & \approx e^{\frac{\tau}{2}\left(\mathcal{H}_{S}+\mathcal{H}_{F}\right)} e^{\tau \mathcal{U}_{S F}} e^{\frac{\tau}{2}\left(\mathcal{H}_{S}+\mathcal{H}_{F}\right)} \\
& \approx e^{\frac{\tau}{2} \mathcal{H}_{F}} e^{\frac{\tau}{2} \mathcal{H}_{S}} e^{\tau \mathcal{U}_{S F}} e^{\frac{\tau}{2} \mathcal{H}_{S}} e^{\frac{\tau}{2} \mathcal{H}_{F}}
\end{aligned}
$$

which is in the BRIDGE format. ${ }^{3}$ In Eq. $13, S$ and $F$ are coupled by $\mathcal{U}_{S F}, \mathcal{H}_{S}$ is solved by a standard $\phi_{\tau}$-type method and $\mathcal{H}_{F}$ is solved recursively, by applying Eq. 13 with $\tau \rightarrow \tau / 2$ on each recursive call, until there are no more particles in $F$.

The map in Eq. 13 is of 2nd-order, but it can be generalized to an arbitrary order $Y$ and number of stages $2 Z+1$ by introducing the Slow-Fast Drift \& Kick operators, defined as ${ }^{S F} D_{\tau} \equiv e^{\tau\left(\mathcal{H}_{F}+\mathcal{H}_{S}\right)}$ and ${ }^{S F} K_{\tau} \equiv e^{\tau \mathcal{U}_{S F}}$, so that,

$$
\begin{aligned}
S F D K D_{\tau}^{Y Z} \equiv & {\left[\prod_{z=1}^{Z} S F D_{a_{z} \tau} S F K_{b_{z} \tau}\right] S F D_{a_{Z} \tau} S F K_{b_{Z} \tau} S F D_{a_{Z} \tau} } \\
& \times\left[\prod_{z=1}^{Z} S F K_{b_{z^{\prime}} \tau} S F D_{a_{z^{\prime}} \tau}\right],
\end{aligned}
$$

where $z^{\prime}=Z-z$ and $a$ 's and $b$ 's are the order condition coefficients (see Tab. 1). In more practical terms, ${ }^{S F} D_{\tau}$ and ${ }^{S F} K_{\tau}$ are implemented as,

$$
\left\{\begin{array}{l}
{ }^{S F} D_{\tau} \rightarrow{ }^{S F} D K D_{\tau}^{Y Z}(F) D K D_{\tau}^{Y Z}(S) \text { if } F \neq \emptyset, \text { else } D K D_{\tau}^{Y Z}(S), \\
{ }^{S F} K_{\tau} \rightarrow{ }^{S F} K_{\tau}^{P N} \text { if } P N \text { is True, else }{ }^{S F} K_{\tau}^{N}
\end{array}\right.
$$


where $D K D_{\tau}^{Y Z}$ is a generalization of a standard map as in Eq. 6, with $D_{\tau} \equiv e^{\tau \mathcal{T}}$ and $K_{\tau} \equiv e^{\tau \mathcal{U}}$

$$
D K D_{\tau}^{Y Z} \equiv\left[\prod_{z=1}^{Z} D_{a_{z} \tau} K_{b_{z} \tau}\right] D_{a_{Z} \tau} K_{b_{Z} \tau} D_{a_{Z} \tau}\left[\prod_{z=1}^{Z} K_{b_{z^{\prime}} \tau} D_{a_{z^{\prime}} \tau}\right]
$$

and, similarly, $D_{\tau}$ and $K_{\tau}$ are implemented as,

$$
\left\{\begin{array}{l}
D_{\tau} \rightarrow D_{\tau}^{P N} \text { if } P N \text { is True, else } D_{\tau}^{N}, \\
K_{\tau} \rightarrow K_{\tau}^{P N} \text { if } P N \text { is True, else } K_{\tau}^{N}
\end{array}\right.
$$

In Eqs. 15 and 17, symbols with superscript $N$ and $P N$ refers to Newtonian and post-Newtonian operators. They are given explicitly as follows:

$$
\begin{aligned}
& D_{\tau}^{N}: \quad \mathbf{r}_{n+1}=\mathbf{r}_{n}+\tau \mathbf{v}_{n+\frac{1}{2}}, \\
& K_{\tau}^{N}: \quad \mathbf{v}_{n+1}=\mathbf{v}_{n}+\tau \mathbf{F}\left(\mathbf{r}_{n+\frac{1}{2}}\right) \text {, } \\
& D_{\tau}^{P N}: \quad \mathbf{r}_{n+1}=\mathbf{r}_{n}+\tau \mathbf{v}_{n+\frac{1}{2}}, \\
& K_{\tau}^{P N}:\left\{\begin{array}{l}
\mathbf{v}_{n+\frac{1}{2}}=\mathbf{v}_{n}+\frac{\tau}{2} \mathbf{G}\left(\mathbf{r}_{n+\frac{1}{2}}, \mathbf{u}_{n}\right), \\
\mathbf{u}_{n+1}=\mathbf{u}_{n}+\tau \mathbf{G}\left(\mathbf{r}_{n+\frac{1}{2}}, \mathbf{v}_{n+\frac{1}{2}}\right), \\
\mathbf{v}_{n+1}=\mathbf{v}_{n+\frac{1}{2}}+\frac{\tau}{2} \mathbf{G}\left(\mathbf{r}_{n+\frac{1}{2}}, \mathbf{u}_{n+1}\right),
\end{array}\right. \\
& S F K_{\tau}^{N}:\left\{\begin{array}{l}
\mathbf{v}_{n+1}^{S}=\mathbf{v}_{n}^{S}+\tau \mathbf{F}\left(\mathbf{r}_{n+\frac{1}{2}}^{F}\right) \\
\mathbf{v}_{n+1}^{F}=\mathbf{v}_{n}^{F}+\tau \mathbf{F}\left(\mathbf{r}_{n+\frac{1}{2}}^{S}\right)
\end{array}\right. \\
& { }^{S F} K_{\tau}^{P N}:\left\{\begin{array}{l}
\mathbf{v}_{n+\frac{1}{2}}^{S}=\mathbf{v}_{n}^{S}+\frac{\tau}{2} \mathbf{G}\left(\mathbf{r}_{n+\frac{1}{2}}^{F}, \mathbf{u}_{n}^{F}\right), \\
\mathbf{v}_{n+\frac{1}{2}}^{F}=\mathbf{v}_{n}^{F}+\frac{\tau}{2} \mathbf{G}\left(\mathbf{r}_{n+\frac{1}{2}}^{S}, \mathbf{u}_{n}^{S}\right), \\
\mathbf{u}_{n+1}^{S}=\mathbf{u}_{n}^{S}+\tau \mathbf{G}\left(\mathbf{r}_{n+\frac{1}{2}}^{F}, \mathbf{v}_{n+\frac{1}{2}}^{F}\right), \\
\mathbf{u}_{n+1}^{F}=\mathbf{u}_{n}^{F}+\tau \mathbf{G}\left(\mathbf{r}_{n+\frac{1}{2}}^{S}, \mathbf{v}_{n+\frac{1}{2}}^{S}\right), \\
\mathbf{v}_{n+1}^{S}=\mathbf{v}_{n+\frac{1}{2}}^{S}+\frac{\tau}{2} \mathbf{G}\left(\mathbf{r}_{n+\frac{1}{2}}^{F}, \mathbf{u}_{n+1}^{F}\right), \\
\mathbf{v}_{n+1}^{F}=\mathbf{v}_{n+\frac{1}{2}}^{F}+\frac{\tau}{2} \mathbf{G}\left(\mathbf{r}_{n+\frac{1}{2}}^{S}, \mathbf{u}_{n+1}^{S}\right),
\end{array}\right.
\end{aligned}
$$

where $\mathbf{u}$ is an auxiliary velocity, with $\mathbf{u}_{0}=\mathbf{v}_{0}$, and $\mathbf{G} \equiv \mathbf{F}+\mathbf{F}^{P N}$ is the total, Newtonian plus post-Newtonian force. We use post-Newtonian corrections up to order 3.5PN as given in Refs. 6, 7. Notice that these post-Newtonian terms are included following the Auxiliary Velocity Algorithm of Ref. 8, which is explicit and time-symmetric as show in the definition of operator $K_{\tau}^{P N}$ Eq. 19, whereas in Eq. 21 we present its generalization for the case of a Slow-Fast Hamiltonian split. 
Table 1. Symplectic integration algorithms classified by name, integration order, number of stages and time-step scheme. The rightmost column gives the reference for the order condition coefficients of the maps.

\begin{tabular}{|c|c|c|c|c|}
\hline name & order $(Y)$ & stages $(Z)$ & time-step & Ref. \\
\hline c.sia21.dkd / c.sia21.kdk & \multirow{3}{*}{2} & \multirow{3}{*}{1} & constant & \multirow{3}{*}{5} \\
\hline a.sia21.dkd / a.sia21.kdk & & & adaptive & \\
\hline h.sia21.dkd / h.sia21.kdk & & & hierarchical & \\
\hline c.sia22.dkd / c.sia22.kdk & \multirow{3}{*}{2} & \multirow{3}{*}{2} & constant & \multirow{3}{*}{9} \\
\hline a.sia22.dkd / a.sia22.kdk & & & adaptive & \\
\hline h.sia22.dkd / h.sia22.kdk & & & hierarchical & \\
\hline c.sia43.dkd / c.sia43.kdk & \multirow{3}{*}{4} & \multirow{3}{*}{3} & constant & \multirow{3}{*}{5} \\
\hline a.sia43.dkd / a.sia43.kdk & & & adaptive & \\
\hline h.sia43.dkd / h.sia43.kdk & & & hierarchical & \\
\hline c.sia44.dkd / c.sia44.kdk & \multirow{3}{*}{4} & \multirow{3}{*}{4} & constant & \multirow{3}{*}{9} \\
\hline a.sia44.dkd / a.sia44.kdk & & & adaptive & \\
\hline h.sia44.dkd / h.sia44.kdk & & & hierarchical & \\
\hline c.sia45.dkd / c.sia45.kdk & \multirow{3}{*}{4} & \multirow{3}{*}{5} & constant & \multirow{3}{*}{9} \\
\hline a.sia45.dkd / a.sia45.kdk & & & adaptive & \\
\hline h.sia45.dkd / h.sia45.kdk & & & hierarchical & \\
\hline c.sia46.dkd / c.sia46.kdk & \multirow{3}{*}{4} & \multirow{3}{*}{6} & constant & \multirow{3}{*}{10} \\
\hline a.sia46.dkd / a.sia46.kdk & & & adaptive & \\
\hline h.sia46.dkd / h.sia46.kdk & & & hierarchical & \\
\hline c.sia67.dkd / c.sia67.kdk & \multirow{3}{*}{6} & \multirow{3}{*}{7} & constant & \multirow{3}{*}{5} \\
\hline a.sia67.dkd / a.sia67.kdk & & & adaptive & \\
\hline h.sia67.dkd / h.sia67.kdk & & & hierarchical & \\
\hline c.sia69.dkd / c.sia69.kdk & \multirow{3}{*}{6} & \multirow{3}{*}{9} & constant & \multirow{3}{*}{11} \\
\hline a.sia69.dkd / a.sia69.kdk & & & adaptive & \\
\hline h.sia69.dkd / h.sia69.kdk & & & hierarchical & \\
\hline
\end{tabular}

\section{Conclusion}

We have developed a family of hierarchical symplectic integration methods for arbitrary $N$-body systems. All 48 maps include post-Newtonian corrections up to order 3.5PN. Such methods can be applied to any physical system whose time evolution is governed by Eq. 1. Besides that, the hierarchical Hamiltonian splitting on which these maps are based can be used as a meta-algorithm for the hierarchical coupling of different astrophysical domains in numerical simulation codes. An application using such methodology is currently in development and will be presented elsewhere.

\section{Acknowledgments}

The author thanks Prof. H. A. Dottori, Prof. S. O. Kepler and Dr. R. O. Gomes for the support and incentive and acknowledge CAPES and the organizers of the 7th IWARA for the financial support. 


\section{References}

1. V. Springel, Monthly Notices of the Royal Astronomical Society 364, 1105 (Dec. 2005).

2. F. I. Pelupessy, A. van Elteren, N. de Vries, S. L. W. McMillan, N. Drost and S. F. Portegies Zwart, Astronomy and Astrophysics 557, p. A84 (Sep. 2013).

3. M. Fujii, M. Iwasawa, Y. Funato and J. Makino, Publications of the Astronomical Society of Japan 59, 1095 (Dec. 2007).

4. F. I. Pelupessy, J. Jänes and S. Portegies Zwart, New Astronomy 17, 711 (Nov. 2012).

5. H. Yoshida, Physics Letters A 150, 262 (Nov. 1990).

6. Y. Itoh, Physical Review D 69, 064018 (Mar. 2004).

7. Y. Itoh, Physical Review D 80, 124003 (Dec. 2009).

8. C. Hellström and S. Mikkola, Celestial Mechanics and Dynamical Astronomy 106, 143 (Feb. 2010).

9. I. P. Omelyan, I. M. Mryglod and R. Folk, Computer Physics Communications 151, 272 (Apr. 2003).

10. S. Blanes and P. C. Moan, Journal of Computational and Applied Mathematics 142, 313 (May 2002).

11. W. Kahan and R.-C. Li, Mathematics of Computation 66, 1089 (1997). 